\title{
Perfil das Gestantes Usuárias de Álcool/Drogas e os Efeitos na Saúde e Desenvolvimento dos Filhos
}

\author{
Kaynara Maria Chenini Motta \\ Maria Beatriz Martins Linhares* \\ Universidade de São Paulo, Faculdade de Medicina, Ribeirão Preto, SP, Brasil
}

\begin{abstract}
RESUMO
O objetivo do estudo foi revisar artigos sobre prevalência do uso de álcool e/ou drogas ilícitas em gestantes, perfis das usuárias e efeitos do seu uso na saúde e desenvolvimento dos filhos. Os estudos foram selecionados na base de dados Scielo a partir de palavras-chave específicas e critérios de inclusão e exclusão. Foram encontrados 14 artigos. Os achados mostraram prevalências variando de 2,1 a $67,1 \%$ para álcool e 0,6 a 53,8\% de drogas ilícitas nas gestantes. As prevalências dependeram de fatores sociodemográficos e clínicos. O uso abusivo de álcool e drogas ilícitas na gestação apresentaram efeitos negativos na saúde e desenvolvimento dos filhos. A revisão realizada mostra a relevância da implementação de políticas públicas de saúde para prevenção do uso de drogas em gestantes, especialmente as adolescentes.
\end{abstract}

Palavras-chave: gravidez; abuso de álcool; drogas; desenvolvimento da criança.

\section{ABSTRACT \\ Profile of Pregnant Using Alcohol/Drugs and Their Effects on Children's Health and Development}

The aim of the study was to review empirical studies about the prevalence of using alcohol and illicit drugs in pregnants, the profiles of them, and the effects of their using on the children's health and development. The studies were selected through the Scielo database using specific keywords and inclusion and exclusion criteria. Fourteen studies were found. The findings showed prevalence ranging from 2.1 to $67.1 \%$ for alcohol and 0.6 a $53.8 \%$ for illicit drugs in pregnants. This prevalence was dependent of socio-economical and clinical factors. The abusive using of alcohol and illicit drugs during the pregnancy presented negative effects on children's health and development. The present review showed the relevance of the implementation of public health policies for drugs abuse prevention in pregnants, especially adolescents.

Keywords: pregnancy; alcohol abuse; drugs; child development.

O abuso de substâncias tem sido motivo de preocupação mundial com relação à saúde da gestante e de seu filho em diferentes níveis socioeconômicos (Angelucci, 2010; van Gelder et al., 2010; Meyer-Leu, Lemola, Daeppen, Deriaz, \& Gerber, 2011; Hayatbakhsh et al., 2011; De Santis et al., 2011; Behnk \& Smith, 2013; Schauberger, Newbury, Colburn, \& Al-Hamadam, 2014). Recentemente, pesquisas têm alertado para o aumento progressivo e alarmante no consumo de álcool, tabaco, maconha e outras drogas no sexo feminino, especialmente durante a gestação (Angelucci, 2010). As prevalências de uso dessas substâncias por mulheres grávidas variam muito e tem sido difícil estabelecer sua estimativa do fenômeno real (Aliane, 2008).

Quase todas as drogas podem ter efeito sobre o feto (Behnke \& Smith, 2013). Quando se realiza o uso ou abuso de álcool e/ou drogas, essas substâncias podem atravessar a placenta, assim como acontece com o oxigênio, o gás carbônico e a água. $\mathrm{O}$ uso do álcool

* Endereço para correspondência: Maria Beatriz Martins Linhares - linhares@fmrp.usp.br 
na gravidez ainda que em nível moderado afeta negativamente o desenvolvimento do feto acarretando o nascimento com baixo peso (Meyer-Leu et al., 2011). A exposição pré-natal ao álcool e outras drogas pode acarretar problemas, os quais podem ser tratados se a presença do uso de droga for detectada precocemente. A vulnerabilidade no período inicial da gestação é maior, na medida em que o desenvolvimento é mais rápido (Papalia, Olds, \& Feldman, 2006).

No estudo de revisão de Leite, Paumgartten e Koifman (2002) o uso do álcool durante a gravidez foi apontado como causa de alterações nas estruturas craniofaciais podendo estar associado à ocorrência de fenda palatina e lábio leporino. De forma complementar, estudos de revisão indicam que a exposição prénatal ao álcool causa anomalias severas no desenvolvimento do córtex cerebral e o desenvolvimento anormal do cérebro pode resultar em distúrbios funcionais na idade adulta (Aversi-Ferreira \& Nascimento, 2008; Yamaguchi, Cardoso, Torres, \& Andrade, 2008). A Síndrome Alcoólica Fetal (SAF) é a consequência mais séria para o feto após o consumo de álcool durante a gestação e se refere a um conjunto de características e atrasos no desenvolvimento de crianças nascidas de mães que consumiram álcool durante a gravidez (Yamaguchi et al., 2008; Momino, Sanseverino, \& Schüler-Faccini, 2008). De acordo com estes estudos, a SAF é caracterizada por retardo do crescimento pré-natal ou pós-natal, déficit mental, alterações musculoesqueléticas, geniturinárias e cardíacas, anormalidades neurológicas, disfunções comportamentais e atrasos desenvolvimentais. $\mathrm{O}$ fato de ingerir álcool durante a gestação pode gerar na criança dificuldades emocionais, problemas com socialização e comunicação mesmo sem o diagnóstico completo de SAF (Momino et al., 2008).

A prevalência do uso de cocaína e crack têm aumentado drasticamente na população de gestantes durante as últimas décadas (Yamaguchi et al., 2008). Cerca de $10 \%$ das mulheres norte-americanas utilizaram cocaína durante a gestação, ocorrendo parto prétermo ou descolamento prematuro de placenta na grande maioria dessas pacientes, além de outras complicações maternas e perinatais. De acordo com este estudo, o efeito do uso da cocaína no feto envolve os seguintes aspectos: vasoconstrição, insuficiência úteroplacentária, hipoxemia, acidose fetal, além de malformações urogenitais, cardiovasculares e do sistema nervoso central. Além disso, o uso da maconha na fase perinatal apresenta como efeito diminuição da perfusão úteroplacentária, prejudicando o crescimento fetal e levando ao retardo da maturação do sistema nervoso fetal (Yamaguchi et al., 2008).

Diante do exposto, foi possível observar a relevância de se investigar no contexto nacional sobre o tema álcool e drogas ilícitas na gestação e seu impacto. Portanto, o presente estudo teve por objetivo realizar uma revisão sistemática da literatura nacional publicada e indexada sobre a prevalência do uso de álcool e drogas ilícitas em gestantes, perfil sociodemográfico e clínico de gestantes usuárias dessas substâncias, padrão de consumo e as consequências do uso do álcool e drogas ilícitas na saúde e no desenvolvimento dos filhos.

\section{MÉTODO}

A presente revisão bibliográfica foi realizada por meio da seleção de artigos científicos publicados na base de dados Scielo. As palavras chaves foram determinadas por meio de pesquisa nos termos Decs. Assim sendo, as palavras-chaves selecionadas foram: Drogas and Feto; Gestação and Álcool; Gestação and Desenvolvimento and Álcool; Gestação and Desenvolvimento and Drogas; Gestação and Drogas; Gravidez and Álcool; Gravidez and Drogas; Síndrome and Alcoólico and Fetal e Síndrome and Fetal and Álcool. Os critérios de inclusão foram os seguintes: estudos empíricos que investigaram por meio de dados de pesquisas o perfil de gestantes usuárias de álcool e drogas e os efeitos desse uso na saúde e desenvolvimento dos filhos; artigos publicados nos idiomas português, inglês ou espanhol. Os critérios de exclusão, por sua vez foram os seguintes: artigos de revisão, metanálises, comentários, editoriais, estudos de caso ou cartas; estudos que não desenvolveram a mensuração ou investigação do perfil de gestantes usuárias de álcool e drogas e os efeitos desse uso na saúde e desenvolvimento dos filhos; estudos cujos objetivos centravam-se exclusivamente em temas médicos, artigos publicados em língua diferente do português, inglês e espanhol.

A pesquisa foi realizada no mês de fevereiro de 2013 sem limite de tempo antecedente. Inicialmente foram encontrados 246 artigos na base de dados Scielo e excluídos 114 repetidos. Em seguida foram analisados 132 artigos e eliminados 118 artigos de acordo com os seguintes critérios de exclusão: revisões, car- 
tas, editoriais ou estudo de caso $(n=14)$, pesquisa com animais $(n=2)$, estudos psicométricos $(n=1)$, não abordavam o uso de drogas ilícitas e/ou álcool $(n=9)$, não participaram gestantes na amostra $(n=5)$, estudos que tinham outros objetivos $(\mathrm{n}=86)$ e artigo mexicano $(\mathrm{n}=1)$. Foram analisados 14 artigos na presente revisão.

Os artigos foram lidos e fichados individualmente em uma tabela contendo os seguintes itens: autor, ano, objetivo, delineamento do estudo, amostra, variáveis, instrumentos e medidas, coleta de dados, resultados, conclusão e observações. Os artigos foram analisados por dois pesquisadores (autores do estudo) e agrupados em categorias de acordo com os resultados desse fichamento e o objetivo da revisão. Portanto, os resultados serão apresentados de acordo com os objetivos nos seguintes tópicos: prevalência do uso de álcool e drogas em gestantes, perfil sociodemográfico e clínico de gestantes usuárias dessas substâncias, padrão de consumo e consequências do uso de álcool e drogas ilícitas na gestação na saúde e no desenvolvimento dos filhos.

\section{RESULTADOS}

\section{Prevalência do uso do álcool e drogas ilícitas em gestantes}

Com relação à prevalência da ingestão de álcool na gestação foi possível verificar que em adolescentes da Zona Norte da cidade de São Paulo, entre 14 e 24 anos, a prevalência foi de $26,6 \%$ para ingestão de ao menos uma vez durante a gestação, sendo que cerca de 2,8\% fez este uso de forma abusiva. Quanto aos outros tipos de drogas, a prevalência do uso de maconha e cocaína foi de $1,7 \%$ e $0,6 \%$ para uso de drogas injetáveis em adolescentes grávidas (Chalem et al., 2007). Em outro estudo foi observado que $82,5 \%$ das puérperas adolescentes da cidade de Fortaleza (CE), não utilizaram nenhum tipo de droga. Destaca-se que entre as gestantes que relataram uso de drogas e/ou álcool, 1,5\% fizeram uso simultâneo de drogas ilícitas, álcool e cigarro, $5 \%$ de grávidas consumiram álcool, $4,5 \%$ álcool e fumo e $0,5 \%$ fumo e drogas ilícitas (Caminha et al., 2012).

Em entrevista com mulheres, adolescentes e adultas, atendidas na rede pública de saúde da cidade do Rio de Janeiro, 40,6\% reportaram uso de bebida alcoólica durante algum período da gestação, enquanto que $10,1 \%$ relataram ter feito uso do álcool até o final da gestação (Moraes \& Reichenheim, 2007). Entre as puérperas adultas, atendidas em maternidade pública do Rio de Janeiro, com idade igual ou superior a 20 anos, 7,4\% indicaram ter utilizado álcool durante a gestação (Freire, Padilha, \& Saunders, 2009). As bebidas que apareceram como mais consumidas foram: cerveja $(83,9 \%)$ e vinho $(9,7 \%)$. As demais como whisky, cachaça, licor, batida e outros representaram 6,5\% do consumo (Moraes \& Reichenheim, 2007). O uso de álcool também apareceu associado ao fumo, sendo que $31,3 \%$ das gestantes adultas fumantes fizeram uso concomitante de cigarro e álcool (Freire et al., 2009). Durante a gravidez, 18,6\% de 485 puérperas adolescentes e adultas atendidas no Hospital das Clínicas da Universidade Federal de Uberlândia, consumiram outras drogas que não o álcool, entre estas, o tabaco foi consumido por $18,4 \%$, o crack por $3,1 \%$, a maconha por $1,2 \%$ e a cocaína por $0,6 \%$ (Souza, Santos, \& Oliveira, 2012). Foi possível observar que entre as puérperas entrevistadas, por meio do questionário Alcohol Use Disorder Identification Test (AUDIT), no período de 12 a 24 horas após o parto, 23,1\% consumiram bebidas alcoólicas em algum momento após a confirmação da gravidez. Segundo os dados do questionário Cut down, Annoyed by Criticism, Guilty and Eye - opener (CAGE), 13,1\% das gestantes fizeram o uso de álcool. O consumo alcoólico de risco foi mais frequente entre gestantes com escolaridade até o ensino fundamental sendo, $8,8 \%$ versus $3,3 \%$ para o questionário AUDIT e $18,1 \%$ versus $9,6 \%$ para o questionário CAGE (Souza et al., 2012).

É importante ressaltar que todas as gestantes adolescentes e adultas, atendidas no Hospital das Clínicas da Universidade de Uberlândia, avaliadas por meio do questionário AUDIT, Tolerance Cut-down, Annoyed, Eye-opener (T-ACE) e CAGE, foram identificadas como consumidoras de alto risco ou prováveis dependentes. Das gestantes que foram diagnosticadas como consumidoras de risco, 96,3\% tiveram este resultado no questionário T-ACE e $86,9 \%$ no questionário CAGE (Souza et al., 2012). Em outra pesquisa, foram utilizados três questionários que visavam mensurar o uso, abuso ou provável dependência de álcool, sendo estes questionários CAGE, T-ACE e Tolerance Worry Eye-opener Annoyed Cut-down (TWEAK). Entre as 521 mulheres adolescentes e adultas da cidade do Rio de Janeiro, que responderam aos três questionários, $26,7 \%$ foram consideradas consumidoras de alto risco ou provável dependente por ao menos um dos instru- 
mentos e apenas 5,6\% tiveram este mesmo resultado com os três questionários. Somente uma mulher entre as 114 mulheres identificadas pelo T-ACE não foi detectada pelo TWEAK (Moraes \& Reichenheim, 2007).

\section{Perfil sociodemográfico e clínico das gestantes usuárias de álcool e drogas ilícitas}

Com relação aos fatores socioeconômicos e clínicos, os estudos verificaram que os seguintes fatores interferiram no uso de bebidas alcoólicas: baixo nível de escolaridade (Moraes \& Reichenheim, 2007; Souza et al., 2012), idade materna, ter histórico de aborto anterior (Moraes \& Reichenheim, 2007; Freire et al., 2009) ser fumante, ter usado drogas durante a gestação, não ser primigesta (Moraes \& Reichenheim, 2007), ser gestante adolescente ou apresentar história de gravidez na adolescência (Gama, Szwarcwald, \& Leal, 2002), ter pouco apoio social (Moraes \& Reichenheim, 2007; Freire et al., 2009) e não coabitar com o companheiro (Freire, Machado, Melo, \& Melo, 2005; Freire et al., 2009; Moraes \& Reichenheim, 2007; Souza et al., 2012).

O uso nocivo de álcool ou provável dependência foi mais frequente entre as gestantes adolescentes e adultas que não coabitavam com o companheiro, sendo, $6 \%$ para as gestantes que não coabitavam com o companheiro versus $1,7 \%$ para as que coabitavam com seus parceiros (Souza et al., 2012). As mulheres adultas que viviam sem o companheiro tiveram chance quase três vezes maior de ingerir álcool na gestação em comparação às que viviam com companheiro (Freire et al., 2009). Similarmente, a prevalência de casos suspeitos de uso de álcool foi maior entre as mulheres adolescentes e adultas que não viviam com companheiro (Moraes \& Reichenheim, 2007). O uso do questionário CAGE indicou uma relação de 19,5\% para o uso de álcool de gestantes adolescentes e adultas solteiras versus $10,6 \%$ para o uso de álcool de gestantes adolescentes e adultas, que viviam com o companheiro, atendidas no Hospital das Clínicas da Universidade Federal de Uberlândia (Souza et al., 2012). Concluiu-se que o consumo de bebida alcoólica foi significativamente maior nas solteiras em comparação às casadas (Freire et al., 2005).

Outro fator relevante que pareceu influenciar o uso do álcool foi a idade. Por meio do uso dos questionários TWEAK e T-ACE, foi possível observar uma prevalência maior do uso de álcool entre as mulheres com idades mais avançadas, com baixa escolaridade, que se autoclassificaram como de cor não branca, fumantes, que relataram uso de drogas durante a gestação, que tiveram gestações e histórias de abortamentos anteriores e aquelas que referiram níveis mais baixos de apoio social (Moraes \& Reichenheim, 2007). O uso de álcool na gestação não foi associado ao grau de escolaridade materna e condições da moradia quando as participantes dessa amostra não eram portadoras de enfermidades crônicas, de gestação de feto único, peso pré-gestacional conhecido ou medido até o final da $13^{\mathrm{a}}$ semana gestacional, com acesso à assistência pré-natal e com informação acerca do uso de álcool e cigarro na gestação (Moraes \& Reichenheim, 2007).

Adolescentes grávidas ou mulheres que referiram gravidez na adolescência apresentaram prevalência no uso de alguma droga na gestação de 1,2\% e 1,6\% respectivamente, enquanto que o índice foi de $0,2 \%$ para mulheres com idade entre 20 e 34 anos e que não engravidaram na adolescência (Gama et al., 2002). É importante ressaltar que apesar de a amostra do estudo ter apresentado baixo relato de ingestão de drogas, em relação aos indicares de estilo de vida, o grupo de puérperas de 20-34 anos com experiência de gestação na adolescência foi o que apresentou os piores resultados, com maior prevalência de abortos anteriores, consumo de cigarro e de drogas ilícitas na gestação. Esses achados confirmam a hipótese de se tratar de um grupo mais vulnerável no que se refere ao aspecto do cuidado com sua própria saúde e do seu bebê (Gama et al., 2002).

Adicionalmente, foi possível observar que das 50 puérperas adolescentes e adultas (14 e mais de 35 anos) com resultado positivo para o questionário CAGE, que avaliou o alcoolismo em período anterior à gravidez, 30 usaram álcool após o diagnóstico de gravidez. As demais 23 mulheres que apresentaram CAGE negativo no período anterior à gravidez, responderam positivamente a esse questionário no período gestacional (Souza et al., 2012).

No consumo de álcool e drogas ilícitas, além das características sociodemográficas das usuárias, foi relevante analisar o perfil clínico das amostras. Foi possível verificar que $7,4 \%$ das mulheres adultas com idade igual ou superior a 20 anos, residentes no Rio de Janeiro, relataram uso de álcool durante a gestação. O 
perfil clínico dessas mulheres indicou associação com idade materna e história de aborto, sendo que as com idade superior a 35 anos e as com história de aborto foram as que apresentaram maior chance de uso de bebidas alcoólicas no período gestacional (Freire et al., 2009). A história de aborto prévio associado ao resultado positivo no questionário T-ACE, o qual avaliou a ingestão alcoólica, não foi confirmada na amostra de 150 mulheres residentes da cidade de $\mathrm{Ri}$ beirão Preto (Freire et al., 2005).

Em uma amostra de gestantes adolescentes com idade entre 12 a 19 anos, da cidade do Rio de Janeiro, foi possível observar que o número de gestantes que informaram o uso de drogas ilícitas foi pequeno, porém significativamente maior nas adolescentes que receberam nenhuma ou pouca atenção pré-natal $(53,8 \%)$. Quanto ao consumo de bebida alcoólica a diferença também é significativa sendo que $24,6 \%$ relataram ingestão de álcool durante a gestação frente a $15,3 \%$ que não fizeram este uso (Gama, Szwarcwald, Sabroza, Branco, \& Leal, 2004).

Em relação à realização do número precário de consultas na fase pré-natal (0-3), as variáveis que apareceram com efeito significativo foram: filhos nascidos vivos anteriormente, baixo nível de escolaridade materna, consumo de bebidas alcoólicas, uso de cigarros na gestação e tentativa de interromper esta gestação. Pode-se perceber que tanto as adolescentes que fumaram, quanto as que beberam e/ou consumiram drogas na gestação, tiveram um menor número de consultas de pré-natal quando comparadas às demais (Gama et al., 2004).

Foi possível observar que após o diagnóstico da gravidez, houve diminuição nos padrões de consumo alcoólico e aumento no número de gestantes, adolescentes e adultas, abstinentes independentemente do número de consultas pré-natais. No entanto, entre todas as gestantes, $8,3 \%$ continuaram fazendo consumo considerado de risco, nocivo ou eram prováveis dependentes. O resultado positivo do questionário CAGE, o qual indica consumo de álcool, foi mais frequente entre gestantes que fizeram três ou menos consultas de pré-natal $(33,3 \%)$, do que entre as que fizeram quatro ou mais consultas $(11,6 \%$ ) (Souza et al., 2012).

Quanto ao perfil clínico para transtornos psiquiátricos, foi possível verificar que das 450 mulheres adolescentes e adultas, entrevistadas na cidade de
Ribeirão Preto, 38,2\% das gestantes apresentaram suspeita de transtorno psiquiátrico, apresentando resultado positivo no Questionário de Morbidade Psiquiátrica (QMPA). De acordo com os critérios da CID-10, foram encontradas $9,1 \%$ gestantes com diagnóstico de consumo de álcool, das quais 6,0\% apresentaram diagnóstico de uso nocivo e $3,1 \%$ síndrome de dependência ao álcool (Pinheiro, Laprega, \& Furtado, 2005). Essas gestantes com dependência ao álcool apresentaram maior média de sintomas no QMPA, ou seja, maior intensidade de problemas emocionais quando comparadas às gestantes que não tiveram diagnóstico de consumo de álcool. Além disso, essas gestantes apresentaram maior média de pontuação na subescala ansiedade, depressão e álcool do QMPA, quando comparadas às demais gestantes que não tinham diagnóstico de consumo de álcool. Apesar da maior média de pontuação na subescala ansiedade do QMPA apresentada pelas gestantes que faziam uso de álcool, tal resultado não alcançou significância estatística (Pinheiro et al., 2005).

Em pesquisa com adolescentes grávidas, de baixa renda, com idade entre 11 e 19 anos atendidas em um hospital público da cidade de São Paulo, foi observada a prevalência de uso de maconha e/ou cocaína no último trimestre de gravidez em $6 \%$ das pacientes, sendo que $4 \%$ relataram o uso de maconha, $1,7 \%$ uso de cocaína e 0,3\% uso de ambas as drogas (Mitsuhiro, Chalem, Barros, Guinsburg, \& Laranjeira, 2006). Neste estudo observou-se que $27,6 \%$ desta amostra tiveram ao menos um transtorno psiquiátrico, sendo de 12,9\% para Depressão, $10 \%$ para Transtorno Estresse Pós-Traumático, 5,6\% para Transtorno de Ansiedade, $3,5 \%$ para Transtornos Psicóticos, 1,9\% para Somatização, 1,2\% para Transtorno Bipolar, 0,3\% para Transtorno Dissociativo e 0,3\% Transtorno Alimentar (Mitsuhiro et al., 2006).

O uso de álcool na gestação não mostrou associação com o número de gestações, o planejamento gestacional, o estado nutricional materno pré-gestacional, a adequação do ganho de peso gestacional, a assistência nutricional pré-natal, assim como intercorrências durante a gestação (Freire et al., 2009).

\section{Padrão de consumo de álcool e drogas ilícitas em gestantes}

Com relação ao padrão de consumo no que se refere à quantidade do uso de álcool, foi possível mensu- 
rar pelo AUDIT que $43 \%$ das gestantes adolescentes e adultas relataram beber de 2 a 4 vezes por mês, 15,8\% fizeram a ingestão de álcool de 2 a 3 vezes por semana e $6,1 \%$ quase diariamente durante todo o período gestacional (Souza et al., 2012). Sobre a quantidade do consumo de álcool em uma ocasião típica, 8,8\% gestantes adolescentes e adultas referiram beber de 5 a 6 doses, $4,4 \%$ de 7 a 9 e 8,8\%, 10 ou mais doses, totalizando $22 \%$ gestantes que relataram episódio de binge drinking ou abuso agudo de bebidas alcoólicas (cinco ou mais doses em uma ocasião) (Souza et al., 2012). Durante a gravidez, 11,4\% gestantes adolescentes e adultas tiveram a percepção de não conseguir parar de beber uma vez que tivessem iniciado, sendo que para $3,5 \%$ delas isso ocorreu menos que mensalmente, para 3,5\% mensalmente, $2,6 \%$ semanalmente e $1,8 \%$ quase diariamente (Souza et al., 2012). Observou-se ainda que $9,6 \%$ deixaram de fazer alguma atividade habitual durante a gravidez devido ao consumo de álcool, e 7,1\% disseram precisar beber pela manhã para recuperar-se de uma bebedeira anterior (Souza et al., 2012).

Destaca-se que, quanto ao sentimento dessas gestantes adolescentes e adultas, 37,7\% destas relataram que sentiam remorso depois de beber, sendo que para $14,9 \%$ delas isso ocorreu menos que mensalmente, $10,5 \%$ mensalmente, $8,8 \%$ semanalmente e $3,5 \%$ quase diariamente. Cerca de $2,7 \%$ das mulheres disseram que não conseguiram se lembrar do que aconteceu na noite anterior pelo fato de ter bebido, isto ocorreu em $0,9 \%$ gestantes mensalmente e em $1,8 \%$ gestantes semanalmente. Em decorrência da ingestão alcoólica, $6,1 \%$ relataram ter ferido alguém ou ter sido ferida. Somente $43 \%$ foram criticadas pelo consumo de álcool durante a gravidez ou foram orientadas a interromper a ingestão alcoólica (Souza et al., 2012).

\section{Consequências do uso de álcool e drogas ilícitas na gestação na saúde e no desenvolvimento dos filhos}

O uso abusivo de álcool e/ou drogas ilícitas na gestação apresentaram efeitos negativos na saúde e desenvolvimento dos filhos, indicando impacto quanto aos parâmetros antropométricos do recém-nascido (Freire et al., 2005; Silva, Quevedo, Silva, Oliveira, \& Pinheiro, 2011) e pior desempenho dos bebês na Avaliação da Prontidão do Prematuro para Início da avaliação Oral (IAPPIAO) (Gasparin, Silveira, Garcez, \& Levy, 2012). Quanto a este efeito na adolescência, observou-se que adolescentes cujas mães tinham usado álcool durante a gestação apresentaram mais risco para experimentar álcool (Noal, Menezes, Araújo, \& Hallal, 2010).

Em relação à associação entre o abuso de álcool durante a gestação e o baixo peso ao nascer, foi possível observar que entre as mulheres adolescentes e adultas que realizaram acompanhamento pré-natal pelo SUS na cidade de Pelotas (RS) 2,1\% foram avaliadas como consumidoras de álcool e 26,3\% dessas mães tiveram bebês com baixo peso (Silva et al., 2011). Pode-se constatar que a chance de um bebê nascer com baixo peso foi quatro vezes maior entre aqueles cujas mães abusaram de álcool durante a gestação (Silva et al., 2011).

Os parâmetros antropométricos (peso, comprimento e perímetro cefálico) dos recém-nascidos de uma maternidade de Ribeirão Preto (SP) foram reduzidos, quando constatado o uso ou abuso de álcool durante a gestação pelo questionário T-ACE, em comparação com os recém-nascidos em cujas mães não foi constatado o uso ou abuso de álcool utilizando o mesmo questionário (Freire et al., 2005). Observou-se redução de 109 gramas no peso, 1,5 centímetros no comprimento e 0,42 centímetros no perímetro cefálico nos recém-nascidos que suas mães fizeram uso de álcool na gravidez. Não foi observado nenhum caso de anomalia fetal no grupo dos bebês que as mães relataram uso de álcool. Embora sem significado estatístico, os dados desta pesquisa indicaram maior sensibilidade aos efeitos fetais do álcool nos recém-nascidos do sexo feminino, sendo que nestas crianças a redução de peso chegou a 186 gramas (Freire et al., 2005). Em outra pesquisa, realizada com mulheres adultas atendidas em uma maternidade pública do Rio de Janeiro, entre 1999 a 2006 não constatou associação entre o uso de álcool e baixo peso ao nascer e uso de álcool e nascimento prematuro (Freire et al., 2009).

Em pesquisa com recém-nascidos a termo, filhos de mães usuárias de crack e/ou cocaína, foi possível observar que houve pior desempenho destes bebês na IAPPIAO (Gasparin et al., 2012). No entanto, não houve associação entre o uso de drogas/álcool e problemas auditivos (Seixas, Fasolo, \& Roberto, 1993). Quanto ao uso de álcool durante a gestação e seus efeitos no período da adolescência do filho, foi possível observar que esses adolescentes, cujas mães ingeriram álcool na gestação, apresentaram cerca de $60 \%$ mais risco de experimentar álcool quando comparados 
àqueles adolescentes cujas mães não fizeram uso de álcool na gravidez (Noal et al., 2010).

\section{DISCUSSÃO}

Com base nos achados da revisão realizada, primeiramente, foi possível observar que, quanto às prevalências da ingestão de álcool por adolescentes, de ao menos uma vez durante a gestação, houve uma variação entre 5\% (Caminha et al., 2012) e 26,6\% (Chalem et al., 2007). É importante ressaltar o fato de que o estudo que indica a prevalência maior foi realizado em um hospital de nível terciário, de referência para gestações de alto risco, o que pode resultar em uma maior prevalência em relação às amostras da população em geral. As prevalências encontradas nos estudos brasileiros foram semelhantes às encontradas em uma amostra de gestantes americanas (Meyer-Leu et al., 2011) e italianas (De Santis, 2011). Porém, observou-se no estudo de Meyer-Leu et al. (2011) que a quantidade do uso pode impactar diretamente nas prevalências encontradas, sendo $30,8 \%$ para menos de dois copos por semana, $7,9 \%$ para uso moderado de dois a quatro copos por semana e $0,9 \%$ com alto nível igual ou maior que cinco copos. A quantidade do uso, portanto, deve ser levada em conta na análise para melhor compreensão dos dados sobre prevalência do uso de álcool na gestação.

Houve uma prevalência similar entre os estudos quanto ao uso exclusivo de drogas ilícitas ou combinadas com o álcool. Em gestantes adolescentes, 1,7\% relataram o uso de maconha e cocaína (Chalem et al., 2007), 0,6\% referiram-se como usuárias de drogas injetáveis (Chalem et al., 2007), 0,5\% relataram uso de drogas ilícitas concomitante com o álcool (Caminha et al., 2012). Além disso, apenas 2,8\% das adolescentes avaliadas indicaram ingestão alcoólica de forma abusiva (Chalem et al., 2007). Os achados dos estudos brasileiros foram semelhantes ao do estudo de van Gelder et al. (2010), em que o uso de drogas ilícitas associou-se ao uso de álcool e tabaco em uma amostra de gestantes americanas.

Destaca-se que, quando avaliadas apenas as gestantes adultas, a incidência do uso do álcool foi menor em comparação a uma amostra de gestantes adolescentes e adultas. Verificou-se que $7,4 \%$ das gestantes adultas relataram uso de álcool durante algum período da gestação (Freire et al., 2009), enquanto que 40,6\% das gestantes adolescentes e adultas indicaram o uso nesta mesma periodicidade e $10,1 \%$ das gestantes adolescentes e adultas fizeram o uso de álcool durante toda a gestação (Moraes \& Reichenheim, 2007). O fato de a gestante ser jovem é um fator de risco importante para o uso de drogas, como também foi verificado por van Gelder et al. (2010), em uma amostra de gestantes da população americana; as gestantes mais jovens ( $<24$ anos) apresentaram maior prevalência no uso de drogas ilícitas do que as mais gestantes mais velhas.

Pode-se verificar também que apenas um estudo avaliou o tipo de bebida que eram consumidas por gestantes adolescentes e adultas, indicando que as maiores prevalências eram de consumo de cerveja seguida pelo consumo de vinho (Moraes \& Reichenheim, 2007). Quanto ao uso de drogas ilícitas um estudo buscou avaliar esta prevalência diferenciando os tipos de droga, sendo observado que o crack apresenta uma prevalência de $3,1 \%$, a maconha de $1,2 \%$ e cocaína 0,6\% (Souza et al., 2012). É importante destacar o fato de que a prevalência do consumo de bebida alcoólica aparece com maior prevalência em comparação às drogas ilícitas, o que pode ser devido a uma possível omissão quanto ao uso das demais drogas. No Brasil, a bebida alcoólica, principalmente a cerveja, é algo aceito socialmente, podendo este fato vir a facilitar o uso desta droga e até mesmo o relato do seu consumo. Em relação às drogas ilícitas como o crack, maconha e cocaína, o seu uso não é aceito pela maior parte da sociedade, devido a sua ilegalidade, com isso o relato do consumo destas pode ser subestimado.

As prevalências do uso de drogas ilícitas dos estudos brasileiros são semelhantes à prevalência de 3,6\% encontrada no estudo de van Gelder et al. (2010), com amostra de gestantes americanas adolescentes e adultas, avaliadas por meio de entrevista por contato telefônico. No entanto, o estudo de Schauberger et al. (2014) encontrou prevalências superiores às supracitadas em gestantes, $7 \%$ para uso da maconha e 6,5\% para opioides. Destaca-se que neste estudo as drogas ilícitas foram medidas diretamente na urina e não por meio do relato das usuárias, o que aumenta a confiabilidade da medida e consequentemente pode aumentar a identificação do uso. Deve-se salientar, portanto, que as prevalências podem variar em função do tipo de amostra (gestantes adolescentes ou adultas) da fonte e do local de coleta (população, centros clínicos de referência), forma de obtenção dos dados (entrevista face a face ou por telefone, questionários, exame do 
sangue ou urina), assim como o tipo de instrumento utilizado para avaliar o uso de drogas lícitas ou ilícitas.

Com relação ao uso de instrumentos de avaliação validados para a população brasileira, os questionários AUDIT, T-ACE, TWEAK e CAGE buscam detectar o uso de risco ou provável dependência de álcool. Em gestantes adolescentes e adultas, verificou-se a mesma alta prevalência e padrão do uso de álcool como consumidoras de alto risco ou de serem prováveis dependentes, tanto medido por meio do AUDIT quanto pelo T-ACE e CAGE (Souza et al., 2012). No entanto, resultados não tão consistentes foram encontrados ao se utilizar o CAGE, T-ACE e TWEAK para avaliar o uso, abuso ou provável dependência do álcool (Moraes $\&$ Reichenheim, 2007). Foi verificado neste estudo que $26,6 \%$ das mulheres foram consideradas consumidoras de alto risco ou provável dependente por pelo menos um dos instrumentos e apenas 5,6\% tiveram este mesmo resultado com os três questionários.

Todos os questionários citados acima possuem a padronização para a população brasileira, porém entre eles destacam-se principalmente dois, os questionários AUDIT e CAGE. O AUDIT é um questionário recomendado pelo Ministério da Saúde, sendo uma das medidas mais empregadas mundialmente para a identificação de grupos de risco e rastreamento do uso inadequado de álcool em amostras clínicas e população geral (Santos, Gouveia, Fernandes, Souza, \& Grangeiro, 2012). De acordo com o Roteiro de Procedimentos Básicos do Centro de Promoção da Saúde CPS (Serviço de Clínica Médica Geral do Hospital das Clínicas da Faculdade de Medicina de Ribeirão Preto - Universidade de São Paulo, 2011) o instrumento CAGE tem boa especificidade e sensibilidade para identificar casos de pessoas que apresentam problemas com a ingestão de álcool. Este questionário visa diagnosticar a Síndrome de Dependência do Álcool por meio de um método de rápida e fácil aplicação, sem questões intimidadoras, com sensibilidade e especificidade comprovadas (Amarala \& Malbergier, 2004). O questionário AUDIT e T-ACE além de avaliar o uso de risco ou provável dependência, avaliam também em suas questões a quantidade de álcool consumida, sendo assim, possuem uma particularidade importante, pois para que a quantidade de doses ingeridas seja considerada prejudicial o resultado do instrumento é comparado de acordo com os resultados padronizados e não depende da percepção da pessoa quanto ao seu consumo alcoólico.
Ainda em relação ao uso de instrumentos, foi possível notar que quanto à avaliação do uso de drogas ilícitas, nos estudos analisados não foram utilizados instrumentos padronizados que indicavam o uso dessas drogas. Para investigar o uso de drogas na população pode ser utilizado um questionário proposto pela World Health Organization (1981) e adaptado para a realidade brasileira. Além disso, o uso de medidas diretas da substância no sangue e na urina pode aumentar a confiabilidade dos resultados, pois os relatos podem informar o uso de forma subestimada.

Alguns fatores socioeconômicos e clínicos parecem interferir no uso de álcool e drogas ilícitas durante a gestação. Ao analisar os estudos, foi possível observar que a maioria delas buscou avaliar a questão de coabitar ou não com o companheiro, seguido pela análise da variável relacionada à escolaridade, idade materna e história de aborto anterior à gestação. As variáveis menos abordadas foram: ser fumante, uso de outras drogas além do álcool durante a gravidez, não ser primigesta, autoclassificação de cor não branca, história de gravidez na adolescência. A elaboração de um perfil das gestantes usuárias de álcool e/ou drogas ilícitas é de suma importância, pois contribui para conhecer os fatores de risco e proteção e a população que comumente está envolvida nesta situação e dessa forma realizar a implantação de programas sociais que visem à prevenção do uso de álcool e drogas durante e após a gestação. Um fator de proteção observado como significativo a partir da análise dos estudos é a situação da gestante coabitar com o companheiro, constituindo uma rede de apoio social de proteção. $\mathrm{O}$ apoio familiar tem um papel importante em situações de crise e eventos negativos da vida o que pode colaborar para amenizar ou reduzir o impacto das adversidades (Silva, Lunardi, Lunardi Filho, \& Tavares, 2005; Walsh, 2005). Neste sentido seria relevante promover esta forma de apoio às gestantes para diminuir o uso de álcool e/ou drogas ilícitas, além de medidas de intervenção psicoeducativas.

Em relação ao padrão de consumo de álcool, apenas um estudo (Souza et al., 2012) buscou abordar este tema, especificando a quantidade de doses ingeridas durante a gestação, a percepção dessas gestantes frente às dificuldades de parar de beber e os seus sentimentos após a ingestão de álcool. Não foram encontradas pesquisas que buscaram mensurar o padrão de consumo com outras drogas, como por exemplo, a maconha, cocaína e o crack, drogas estas utilizadas em grande escala hoje em dia. O padrão de consumo e 
o tipo de droga utilizada, se exclusiva ou combinada, têm sido aspectos considerados nos estudos internacionais para melhor compreensão do fenômeno do uso e abuso de substâncias (De Santis et al., 2011; Schauberger et al., 2014).

Quanto ao perfil clínico das gestantes usuárias de álcool, foi possível observar associação entre idade materna e história de aborto em uma amostra de mulheres grávidas do Rio de Janeiro (Freire et al., 2009). Em contrapartida, outro estudo realizado com adolescentes e adultas na cidade de Ribeirão Preto, não apresentou associação do uso de álcool durante a gestação e histórico de aborto. Em situações de perda gestacional, é relativamente grande o risco de aparecerem sintomas depressivos, ansiedade ou pânico, principalmente alguns meses após a perda (Burgoine et al., 2005). A perda do filho durante a gestação traz diversas reações, normalmente sofridas. A mulher pode apresentar desvalorização da autoimagem, pelo sentimento de que seu corpo não pôde funcionar adequadamente durante a gestação ou pela crença de que não é capaz de desempenhar seu papel biológico e conjugal (Stirtzinger, Robinson, \& Stewart, 1999). Segundo Pinheiro et al. (2005) a presença de diagnóstico de uso nocivo ou síndrome de dependência ao álcool relacionou-se à maior intensidade de sofrimento emocional das gestantes. Quanto ao perfil clínico para transtornos psiquiátricos, foi observado associação do uso de maconha e/ou cocaína durante a gestação com depressão, transtorno estresse pós-traumático, transtorno de ansiedade, transtorno psicótico, somatização, transtorno bipolar, transtorno dissociativo e transtorno alimentar (Mitsuhiro et al., 2006).

A atenção pré-natal e puerperal de qualidade e humanizada é primordial para a saúde da mãe e do recém-nascido. $\mathrm{O}$ cuidado à mulher durante a gestação $\mathrm{e}$ no pós-parto deve abranger ações de prevenção e promoção da saúde, além de diagnosticar e tratar de maneira adequada os problemas que possam ocorrer neste período segundo o Manual Técnico - Pré-natal e Puerpério: atenção qualificada e humanizada (Ministério da Saúde, 2005). É no pré-natal que a mulher pode além de realizar exames para garantir a sua saúde e a do bebê receber informações importantes quanto aos hábitos e estilo de vida, como, por exemplo, os efeitos do uso de álcool e/ou drogas ilícitas que quando utilizadas durante a gestação podem trazer efeitos negativos no desenvolvimento e saúde infantil.
O uso de álcool e/ou drogas ilícitas na gestação apresenta efeitos negativos na saúde e desenvolvimento dos filhos. Porém, mesmo frente a este dado, nesta revisão foram encontrados poucos estudos que buscaram avaliar estes efeitos. Apenas sete estudos indicaram o impacto negativo do uso de álcool e/ou drogas ilícitas durante a gestação. Em um artigo de revisão Reseguel, Puccini e Silva (2007) buscaram avaliar os principais fatores de risco associados a alterações no desenvolvimento infantil e entre eles foi abordado o uso de drogas lícitas e ilícitas durante a gestação. Comumente a usuária de droga utiliza mais do que um tipo durante a gestação. Esta característica, associada a múltiplos problemas sociais e nutricionais concomitantes e à ausência de estudos prospectivos com grande número de usuárias, dificulta o reconhecimento dos efeitos adversos do uso de drogas no período gestacional (Reseguel et al., 2007). Estes aspectos parecem ter dificultado a comprovação das diversas drogas como teratogênicas, pois é preciso que sejam verificadas as relações dose-medida entre o uso da droga e o efeito observado após a exposição fetal.

O fator baixo peso do recém-nascido, associado ao uso de álcool durante a gestação apareceu como o fator mais estudado nas pesquisas revisadas neste estudo (Freire et al., 2005; Freire et al., 2009; Silva et al., 2011). Este achado é semelhante ao encontrado no estudo de Meyer-Leu et al. (2011), em que o uso moderado de álcool (dois a quatro copos por semana) durante a gestação, em uma amostra de mulheres suíças, aumentava o risco de os bebês nascerem com baixo peso. Notou-se, no entanto, que não foi encontrado nesta revisão estudo que avaliasse o baixo peso no nascimento associado a drogas ilícitas. A prematuridade e o baixo peso ao nascer, são considerados como fatores de risco neonatal para mortalidade e problemas no desenvolvimento futuro dos recémnascidos (Ayward, 2002; Kilsztajn, Rossbach, Carmo, \& Sugahara, 2003). De acordo com Eickmann, Lira e Lima (2002) o baixo peso ao nascer está significativamente associado a um déficit de desenvolvimento aos 24 meses de idade. Frente a esta realidade, é de suma importância que mais estudos examinem a associação entre o uso de álcool e/ou drogas ilícitas e o nascimento prematuro e de baixo peso, contribuindo para uma melhor compreensão do grau de vulnerabilidade das crianças que se encontram nesta situação. Desta forma, pode-se ter subsídios para elaborar uma rede de apoio e proteção para o desenvolvimento e a saúde das crianças. 
Nos artigos nacionais revisados, apenas um relata pior desempenho dos bebês, nascidos a termo, na IAPPIAO, no caso em que suas mães fizeram uso de crack e/ou cocaína na gestação (Gasparin et al., 2012). Como o uso de drogas ilícitas não é aceito socialmente, o relato do seu uso acaba sendo subestimado e, portanto, não é possível avaliar suas consequências no bebê de maneira realista.

Além disso, apenas um estudo avaliou filhos de mães usuárias de álcool durante a gestação no período da adolescência. Observou-se que estes adolescentes apresentaram risco maior de experimentar bebida alcoólica quando comparados àqueles adolescentes cujas mães não fizeram este uso durante a gravidez (Noal et al., 2010). O método deste estudo foi de follow-up, o qual se caracteriza no acompanhamento da amostra por um período de tempo. Estudos como este são de suma importância para avaliar o desenvolvimento após a infância dos filhos de mães que fizeram uso de álcool e/ou drogas ilícitas durante a gestação. A exposição pré-natal ao álcool causa anomalias severas no desenvolvimento do córtex cerebral e o desenvolvimento anormal do cérebro pode resultar em distúrbios funcionais na idade adulta (Aversi-Ferreira \& Nascimento, 2008; Yamaguchi et al., 2008).

Em relação ao tipo de droga, o álcool foi a única droga pesquisada individualmente (Freire et al., 2005; Pinheiro et al., 2005; Moraes \& Reichenheim, 2007; Noal et al., 2010; Silva et al., 2011; Souza et al., 2012). Os demais estudos realizaram pesquisas abordando a associação do uso de álcool e cigarro (Freire et al. 2009) e drogas ilícitas (Seixas et al., 1993; Gama et al., 2002; Gama et al., 2004; Mitsuhiro et al., 2006; Chalem et al., 2007; Caminha et al., 2012; Gasparin et al., 2012). Frente a este dado, pode-se concluir que mesmo o uso de drogas ilícitas estarem sendo frequentemente abordado, entre as pesquisas analisadas nesta revisão, não existe pesquisas específicas para uma determinada droga como, por exemplo, a cocaína. Este fato pode estar relacionado à questão de o álcool ser uma droga lícita que tem seu uso difundido em quase todo o mundo (Grinfeld, 2009) e que normalmente está associada a outro tipo de droga ilícita ou não.

A presente revisão embora apresente a limitação no que se refere à pesquisa em apenas uma base de dados nacional e teve o limite de tempo até fevereiro de 2013, atendeu ao objetivo proposto inicialmente. Os achados da presente revisão mostraram dados relevantes sobre prevalência e perfil das gestantes usuá- rias de álcool e drogas ilícitas, os quais podem subsidiar estratégias de prevenção e estabelecimento de políticas públicas de saúde nesta área, em especial direcionadas para o grupo de gestantes e adolescentes. Estudos futuros nacionais devem ser direcionados para medidas diretas do uso de tais substâncias, não apenas por relato, assim como para a análise dos efeitos nos filhos de gestantes usuárias de álcool e drogas ilícitas. Além disso, são necessários estudos de revisão sobre estratégias eficazes de prevenção para o uso e abuso de álcool e drogas ilícitas na gestação.

\section{REFERÊNCIAS}

Aliane, P. P. (2008). Uso de álcool na gestação e sua relação com sintomas depressivos no pós-parto (Dissertação de Mestrado) - Universidade de São Paulo - Faculdade de Medicina de Ribeirão Preto.

Amarala, R. A., \& Malbergier, A. (2004). Avaliação de instrumento de detecção de problemas relacionados ao uso do álcool (CAGE) entre trabalhadores da Prefeitura do Campus da Universidade de São Paulo (USP) - Campus Capital. Revista Brasileira de Psiquiatria, 26, 156-163. Doi: 10.1590/ S1516-44462004000300005

Angelucci, M. A. (2010). Exposição ao álcool na gestação e sintomas psiquiátricos na idade escolar (Dissertação de Mestrado). Universidade de São Paulo - Faculdade de Ciências Farmacêuticas de Ribeirão Preto.

Aversi-Ferreira, T. A., \& Nascimento, G. N. L. (2008). The effect of acute and chronic exposure to ethanol on the developing encephalon: a review. Revista Brasileira de Saúde Materna Infantil, 8(3), 241-249. Doi: 10.1590/S1519-38292008000300002

Ayward, G. P. Methodological issues in outcomes studies of at-risk infants (2002). Journal of Pediatric Psychology, 27, 37-45. Doi: 10.1093/jpepsy/ 27.1.37

Behnk, M., \& Smith, V. C. (2013). Prenatal Substance Abuse: Short- and Long-term Effects on the Exposed Fetus. American Academy of Pediatrics, 131, 1009-1024. Doi: 10.1542/peds.2012-3931

Burgoine, G. A., Valkirk, S. D., Romm, J., Edelman, A. B., Jacobson, S. L., \& Jensen, J. T. (2005). Comparison of perinatal grief after dilation and avacuation for labor induction in second trimester terminations for fetal anomalies. American Journal of Obstetrics and Gynecology, 192, 1928-1932. Doi: 10.1016/j.ajog.2005.02.064 
Caminha, N. O., Costa, C. C., Brasil, R. F., Sousa, D. M. N., Freitas, L. V., \& Damasceno, A. K. C. (2012). O Perfil das Puérperas Adolescentes Atendidas em uma Maternidade de Referência de Fortaleza-Ceará. Escola Anna Nery, 16, 486-492. Doi: 10.1590/S1414-81452012000300009

Chalem, E., Mitsuhiro, S. S., Ferri, C. P., Barros, M. C. M., Guinsburg, R., \& Laranjeira, R. (2007). Gravidez na adolescência: perfil sócio-demográfico e comportamental de uma população da periferia de São Paulo, Brasil. Caderno de Saúde Pública, 23, 177-186. Doi: 10.1590/S0102-311X200 7000100019

De Santis, M., De Luca, C., Mappa, I., Quattrocchi, T., Angelo, L., \& Cesari, E. (2011). Smoking, alcohol consumption and illicit drug use in an Italian population of pregnant women. European Journal of Obstetrics \& Gynecology and Reproductive Biology, 159, 106-110. Doi: 10.1016/j.ejogrb.2011. 07.042

Eickmann, S. H., Lira, P. I. C., \& Lima, M. C. (2002). Desenvolvimento mental e motor aos 24 meses de crianças nascidas a termo com baixo peso. Arquivos de Neuro-Psiquiatria, 60, 748-754. Doi: 10.1590/S0004-282X2002000500013

Freire, T. M., Machado, J. C., Melo, \& E. V., Melo, D. G. (2005). Efeitos do Consumo de Bebida Alcoólica Sobre o Feto. Revista Brasileira de Ginecologia e Obstetrícia, 27, 376-381. Doi: 10.1590/ S0100-72032005000700002

Freire, K., Padilha, P. C., \& Saunders, C. (2009). Fatores associados ao uso de álcool e cigarro na gestação. Revista Brasileira de Ginecologia e Obstetrícia, 31, 335-341. Doi: 10.1590/S0100-7203200 9000700003

Gama, S. G. N., Szwarcwald, C. L., \& Leal, M. C. (2002). Experiência de gravidez na adolescência, fatores associados e resultados perinatais entre puérperas de baixa renda. Caderno de Saúde Pública, 18, 153-161. Doi: 10.1590/S0102-311X 2002000100016

Gama, S. G. N., Szwarcwald, C. L., Sabroza, A. R., Branco, V. C., \& Leal, M. C. (2004). Fatores associados à assistência pré-natal precária em uma amostra de puérperas adolescentes em maternidades do Município do Rio de Janeiro, 1999-2000. Caderno de Saúde Pública, 20, 101-111. Doi: 10.1590/S0102-311X2004000700011
Gasparin, M., Silveira, J. L., Garcez, L. W., \& Levy, B, S. (2012). Comportamento Motor Oral e Global de Recém-nascidos de Mães Usuárias de Crack e/ou Cocaína. Revista da Sociedade Brasileira de Fonoaudiologia, 17, 459-463. Doi: 10.1590/S151680342012000400016

Grinfeld, H. (2009). Consumo nocivo de álcool durante a gravidez. In: A. G. Andrade \& J. C. Anthony (Eds.) Álcool e suas consequências: uma abordagem multiconceitual (pp. 179-199) Barueri: Manole. Doi: 10.1590/S0034-89102003000300007

Kilsztajn, S., Rossbach, A., Carmo, M.S., \& Sugahara, G. T. (2003). Prenatal care, low birthweight and prematurity in São Paulo State, Brazil. Revista de Saúde Publica, 37, 303-10. Doi: 10.1590/S003489102003000300007

Leite, I. C. G., Paumgartten, F. J. R., \& Koifman, S. (2002). Chemical Exposure During Prenancy and Oral Clefts in Newborns. Caderno de Saúde Pública, 18, 17-31. Doi: 10.1590/S0102-311X2002000 100003

Meyer-Leu, Y., Lemola, S., Daeppen, J. B., Deriaz, O., \& Gerber, S. (2011). Association of Moderate Alcohol Use and Binge Drinking During Pregnancy with Neonatal Health. Alcoholism: Clinical and Experimental Research, 35(9), 1669-1677. Doi: 10.1111/j.1530-0277.2011.01513.x

Ministério da Saúde. Secretaria de Atenção à Saúde. Departamento de Ações Programáticas Estratégicas. Área Técnica de Saúde da Mulher (2005). Pré-natal e Puerpério: atenção qualificada e humanizada - manual técnico - Brasília: Ministério da Saúde. Retirado de http://bvsms.saude.gov.br/bvs/ publicacoes/manual_pre_natal_puerperio_3ed.pdf

Mitsuhiro, S. S., Chalem, E., Barros, M. M., Guinsburg, R., \& Laranjeira, R. (2006). Teenage pregnancy: use of drugs in the third trimester and prevalence of psychiatric disorders. Revista Brasileira de Psiquiatria, 28, 122-125. Doi: 10.1590/S151644462006000200009

Hayatbakhsh, M., Kingsbury, A. M., Flenady, V., Gilshenan, K. S., Hutchinson, D. M., \& Najman, J. M. (2011). Drug and Alcohol Review, 30, 181-187. doi: 10.1111/j.1465-3362.2010.00214.x

Momino, W., Sanseverino, M. T., \& Schüler-Faccini, L. (2008). Prenatal alcohol exposure as a risk factor for dysfunctional behaviors: the role of the pediatrician. Jornal de Pediatria, 84, 76-79. Doi: 10.1590/S0021-75572008000500011 
Moraes, C. L., \& Reichenheim, M. E. (2007). Rastreamento de uso do álcool por gestantes de serviços públicos de saúde do Rio de Janeiro. Revista de Saúde Pública, 41, 695-703. Doi: 10.1590/S003489102007000500002

Noal, R. B., Menezes, A. M. B., Araújo, C. L., \& Hallal, P. C. (2010). Experimental use of alcohol in early adolescence: the 11-year follow-up of the 1993 Pelotas (Brazil) birth cohort study. Caderno de Saúde Pública, 26, 1937-1944. Doi: 10.1590/ S0102-311X2010001000010

Papalia, D. E., Olds, S. W., \& Feldman, R. D. (2006). Concebendo uma nova vida. In: D. E. Papalia; S. W. Olds \& R. D. Feldman (Eds.), Desenvolvimento Humano (pp. 95-124). Porto Alegre: Artmed.

Pinheiro, S. N., Laprega, M. R., \& Furtado, E. F. (2005). Morbidade Psiquiátrica e Uso de Álcool em Gestantes Usuárias do Sistema Único de Saúde. Revista de Saúde Pública, 39, 593-598. Doi: 10.1590/S0034-89102005000400012

Reseguel, R., Puccini, R. F., \& Silva, E. M. K. (2007). Fatores de risco associados a alterações no desenvolvimento da criança/ Risk factors associated with developmental abnormalities in children Factores de riesgo asociados a alteraciones de desarrollo infantile. Pediatria, 29, 117-128. Doi: 10.1590/S15 16-31802008000100002

Santos, W. S., Gouveia, V. V., Fernandes, D. P., Souza, S. S. B., \& Grangeiro, A. S. M (2012). Alcohol Use Disorder Identification Test (AUDIT): explorando seus parâmetros psicométricos. Jornal Brasileiro de Psiquiatria, 61(3), 117-123. Doi: 10.1590/ S0047-20852012000300001

Schauberger, C. V., Newbury, E. J., Colburn, J. M., \& Al-Hamadam, M. (2014). Prevalence of illicit drug use in pregnant women in a Wisconsin private practice setting. American Journal of Obstetrics \& Gynecology, 211(3), 255e1-255e4. Doi: 10.1016/ j.ajog.2014.03.023.

Seixas, R. R., Fasolo, M. I., \& Roberto, N. M. (1993). Avaliação Auditiva Objetiva Através de Potenciais Evocados. Arquivos de Neuro-psiquiatria, 51(4), 433-440 Doi: 10.1590/S0004-282X19930004 00002

Serviço de Clínica Médica Geral HCFMUSP. Prof. Dr. Mílton de Arruda Martins. Centro de Promoção da Saúde - CPS (2011). Roteiro de procedi- mentos básicos. São Paulo: Junior, M. F., Camargo, C. Retirado de http://medicina.fm.usp.br/gdc/ docs/cgp_42_CPS\%20-\%20APOSTILA\%202011. pdf

Silva, M. R. S., Lunardi, V. L., Filho, W. D. L., \& Tavares, K. O. (2005). Resiliência e promoção da saúde/Resilience and health promotion la resiliencia y la promoción de la salud. Texto \& Contexto Enfermagem, 14, 95-102. Doi: 10.1590/S010407072005000500012

Silva, I., Quevedo, L. A., Silva, R. A., Oliveira, S. S., \& Pinheiro, R. T. (2011). Associação entre abuso de álcool durante a gestação e o peso ao nascer. Revista de Saúde Pública, 45, 864-869 Doi: 10.1590/S0034-89102011005000062

Souza, L. H. R. F., Santos, M. C., \& Oliveira, L. C. M. (2012). Padrão do consumo de álcool em gestantes atendidas em um hospital público universitário e fatores de risco associados. Revista Brasileira de Ginecologia e Obstetrícia, 34, 296-303. Doi: 10.1590/S0100-72032012000700002

Stirtzinger, R., Robinson, G.E., \& Stewart, D. (1999). Parameters of grieving in spontaneous abortion. International Journal of Psychiatry in Medicine, 29, 235-249. Doi: 10.2190/UDW4-2EAG-1RTY-D1Y4

van Gelder, M. M. H. J., Reefhuis, J., Caton, A. R., Werler, M. M., Druschel, C. M., \& Roeleveld, N. (2010). Characteristics of pregnant illicit drug users and associations between cannabis use and perinatal outcome in a population-based study. Drug and Alcohol Dependence, 109, 243-247. Doi: 10.1016/ j.drugalcdep.2010.01.007

Walsh, F. (2005). Fortalecendo a resiliência familiar. São Paulo: Roca.

World Health Organization (1981). Drug use among non-student youth. Geneva, Suíça: Smart, R.G., Arif, A.; Hughes, P.; Medina-Mora, M. E.; Navaratnam, V.; Varma, V. K.; Wadud, K. A. Retirado de http://whqlibdoc.who.int/offset/WHO_OFF SET_60.pdf

Yamaguchi, E. T., Cardoso, M. M. S. C., Torres, M. L. A., Andrade, A. G. (2008). Drogas de Abuso e Gravidez. Revista de Psiquiatria Clínica, 35, 44-47. Doi: 10.1590/S0101-60832008000700010.

\footnotetext{
Recebido em: 23/03/2014 Última revisão em: 10/10/2014 Aceito em: 10/12/2014
} 